\title{
Plasmon Resonances for Biospecific Interactions Sensing
}

\author{
Valeri Lozovski, Margarita Razumova
}

Institute of High Technologies, Taras Shevchenko National University of Kyiv, Kyiv, Ukraine

Email address:

v.lozovsk@gmail (V. Lozovski), razumova.m@gmail.com (M. Razumova)

\section{To cite this article:}

Valeri Lozovski, Margarita Razumova. Plasmon Resonances for Biospecific Interactions Sensing. Journal of Photonic Materials and Technology. Vol. 1, No. 2, 2015, pp. 33-39. doi: 10.11648/j.jmpt.20150102.13

\begin{abstract}
An implementation of plasmon resonances in nanocomposite thin films for biosensors is discussed. The effect is studied in the system of modified Au inclusions inside the Teflon matrix. The optical response of the nanocomposite thin film with inhomogeneous distribution of embedded coated Au spherical nanoparticles across the film thickness is analyzed. The absorption profiles are calculated in a case of light incidence normally to the film surface. Their dependences on volume fractions and spatial distributions of inclusion nanoparticles across the film thickness are discussed for some values of the film thickness. The obtained absorption profiles depending on the characteristics of the shell of nanoinclusions allow proposing the optical control method for the biospecific reactions at the surface of modified nanoinclusions.
\end{abstract}

Keywords: Plasmon Resonance, Nanocomposite, Effective Susceptibility, Thin Film, Local Field, Absorption Profile, Biospecific Interaction

\section{Introduction}

The development of life science leads to its becoming more and more interdisciplinary [1-8]. Nowadays, it is already no surprise that a paper devoted to physical or chemical aspects of diagnostics and treatment is published in a medical journal [9], or a paper published in a physical journal deals with the electron properties of DNA systems [10-13] or the physical aspects of virology $[6,7]$. The modern medicine often applies physical methods to diagnostics and treatment of diseases. For instance, the effect of surface plasmon resonance (SPR) [14-16] is widely and successfully applied to diagnostics of some diseases and detecting some molecules or viruses [17-22], study of biological and biochemical processes [23-25], whereas weak electromagnetic radiation in the radio-frequency range has been used for decades as one of the methods to treat a considerable number of diseases. Besides, comparing to other methods of diagnostics, the SPR biosensing allows label-free, high-sensitivity, real-time analysis and flexible system design [19].

The nanocomposite and nanostructured surfaces can be used for optical sensing of biological liquids and control in situ of biospecific interactions. SPR biosensor technology has made substantial advances in terms of both sensor hardware and biospecific coatings. SPR biosensors have been applied for the detection of a variety of chemical and biological analytes. The performance of SPR biosensor technology is continuing to evolve. The advanced SPR sensor platforms are combining with novel biospecific surfaces with high resistance to the nonspecific binding. The robust SPR biosensors will be enabling rapid, sensitive, and specific detection of chemical and biological analytes in complex samples. These biosensors will be useful in numerous important sectors such as medical diagnostics, environmental monitoring, and food safety.

SPR biosensors have become a central tool for characterizing and quantifying biomolecular interactions. The papers [16, 26, 27] review advances in SPR sensor technology and its applications. Main application areas are outlined and examples of applications of SPR sensor technology are presented. The review [27] focuses on SPR sensors employing conventional (unlocalized) surface plasmons propagating along planar structures and their applications for detection of chemical and biological species.

Note that in literature the term "Surface Plasmon Resonance" is widely used both for the case of excitation of surface plasmon waves propagating along the metal surface and for plasmon excitation localized at the nanoparticle. We assume that the usage of the term "surface plasmon resonance" (as "surface plasmon") for plasmon excitation localized at the nanoparticle is not correct. We will refer to these phenomena as "plasmon resonances" or "localized plasmon resonances".

We use the term "plasmon resonance" in a wide sense. Consider the linear response $\mathrm{X}_{i j}(\mathbf{R}, \omega)$ of the system to the external field (effective susceptibility [28]). To calculate the 
local field $E_{i}(\mathbf{R}, \omega)$ at any point $\mathbf{R}$ of the system under consideration one should consider the integral

$$
-(\omega / c)^{2} \int_{V_{p}} d \mathbf{R}^{\prime} G_{i j}\left(\mathbf{R}, \mathbf{R}^{\prime}, \omega\right) \mathbf{X}_{j l}\left(\mathbf{R}^{\prime}, \omega\right) E_{l}^{(\mathrm{ext})}\left(\mathbf{R}^{\prime}, \omega\right),
$$

where $G_{i j}\left(\mathbf{R}, \mathbf{R}^{\prime}, \omega\right)$ is the electrodynamic Green function of the medium in which the nanoparticles are embedded, $E_{l}^{(\text {ext) }}(\mathbf{R}, \omega)$ is the external long-range field, $\omega$ is the frequency of incident external electromagnetic field and $\mathrm{c}$ is the light velocity. The integration is over the volume of particle/particles of the system. Because $X_{i j}(\mathbf{R}, \omega)$ depends on frequency, shape of the particle/particles, and a configuration (relative position of the particles), one can consider three kinds of resonances when the absolute value of $\mathrm{X}_{i j}(\mathbf{R}, \omega)$ strongly increases. First, the induced polarization inside the system is resonantly enhanced at the frequency where the absolute value of the denominator of $\mathrm{X}_{i j}(\mathbf{R}, \omega)$ shows a minimum, limited by the imaginary part of $X_{i j}(\mathbf{R}, \omega)$ describing damping. The resonance frequency is given by the condition that the real part of the denominator vanishes. Herewith the local field is enhanced and the plasmon resonance arises. Second, when at certain frequency the shape of particle(s) causes the occurrence of spatial regions where the local field is strongly enhanced, there is a local field enhancement effect. Third, when at certain frequency and given shapes of the particles, a relative disposition of the particles causes the occurrence of small spatial regions of enhanced local field, the hot spots effect arises which is reflection of so-called configurational resonance. All these effects have a resonant nature and are reflected in the properties of denominator of the $\mathrm{X}_{i j}(\mathbf{R}, \omega)$.

\section{Application of Localized Plasmon Resonance in Biosensors}

Nanocomposite films can be considered as the systems with plasmon resonances. In such systems it can be observed several types of plasmon resonances. Firstly, there are conditions under which the surface (waveguide) modes can propagate along the film. Secondly, the localized at the inclusion particles plasmons can be excited too. Both of the excitation types can be used for sensing. In this chapter we will consider the second type of plasmon excitations - the localized plasmons.

Multifunctional nanocomposites that combine therapeutic, diagnostic, and sensing modalities in a single nanostructure are widely used in theranostics. For example, functionalized gold nanoparticles (GNPs) with controlled geometrical and optical properties are the subject of intensive studies and biomedical applications, including genomics, biosensorics, immunoassays, clinical chemistry, laser phototherapy of cancer cells and tumors, the targeted delivery of drugs, DNA and antigens, optical bioimaging and the monitoring of cells and tissues with the use of state-of-the-art detection systems.
The unique optical properties of GNPs are related to localized plasmonic excitations in metal nanostructures [29] interacting with light. These excitations result in resonance-enhanced local fields and, accordingly, in enhanced optical phenomena.

The theoretical and experimental studies of the optical properties of metal particles with plasmon resonances are presented, for example, in $[29,30]$. Metal spheres, nanorods, spherical and elliptic metal nanoshells are considered. The tuning of plasmon resonances of nanoparticles by varying their size, shape, structure, and dielectric environment as well as modern trends in biomedical applications of nanoparticles with plasmon resonances are discussed.

\section{Linear Response of Thin Nanocomposite Film}

The modern technologies allow obtaining the nanocomposite films with inhomogeneous distribution of inclusions along the film thickness. We consider nanocomposite film at the dielectric substrate with dielectric constant $\varepsilon_{\mathrm{s}}$ (Fig. 1). The identical inclusion particles are supposed to be distributed inhomogeneously along the film thickness and homogeneously in the film plane.

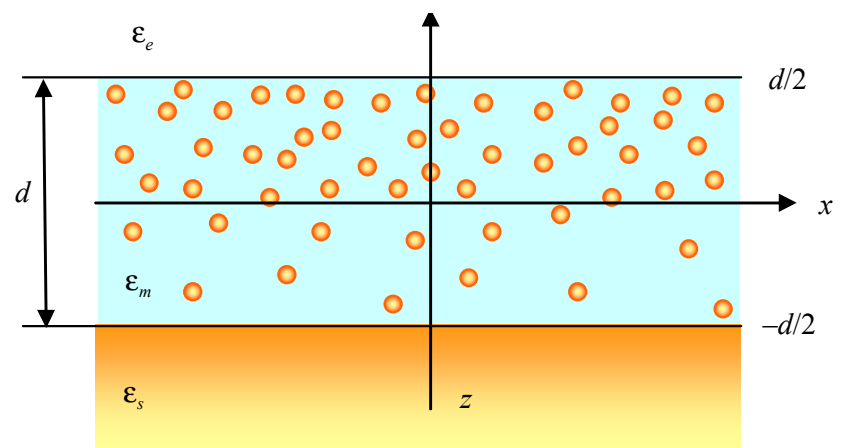

Figure 1. Sketch of the thin film with inhomogeneously along the thickness distributed inclusions.

The effective medium theory can successfully describe the properties of most types of nanocomposites. However, a direct usage of the effective dielectric function of nanocomposite encounters some difficulties within the effective medium theory studying the optical properties of nanocomposite thin films. The problem is that the characteristic length of averaging can be comparable to the film thickness, leading to inability of accounting for the local field inhomogeneities in the film. This means that it is necessary to develop the effective method for calculation of the optical response of the entire film as a whole. The self-consistency can be a method of taking into account the local field inhomogeneity. Such a self-consistent method of effective optical response calculation of the film is proposed in [31].

The starting point is the Lippmann-Schwinger equation of self-consistency [28] averaged over the $N$ inclusion particles positions 


$$
\begin{aligned}
& E_{i}(\mathbf{R}, \omega)=E_{i}^{(0)}(\mathbf{R}, \omega)-k_{0}^{2} \\
& \times\left\langle\sum_{a=1}^{N} \int_{V_{a}} d \mathbf{R}^{\prime} G_{i j}^{(2)}\left(\mathbf{R}, \mathbf{R}^{\prime}, \omega\right) \mathbf{X}_{j l}^{(p)}(\omega) E_{l}\left(\mathbf{R}^{\prime}, \omega\right)\right\rangle,
\end{aligned}
$$

where $E_{i}^{(0)}(\mathbf{R}, \omega)$ is the background (relative to inclusion particles) electric field, $k_{0}=\omega / c, G_{i j}^{(2)}\left(\mathbf{R}, \mathbf{R}^{\prime}, \omega\right)$ is the electrodynamic Green function (the photon propagator), describing the electromagnetic field propagation inside the three layer planar structure with perfect interfaces [32], where both the observation point and the source point are located inside the three-layer system without inclusions. $X_{j l}^{(p)}(\omega)$ is the linear response of the inclusion particle to the local field. The field $E_{i}(\mathbf{R}, \omega)$ in the left and right hand sides of (2) is the local self-consistent field defined at any point inside the system under consideration. Note that using $X_{j l}^{(p)}(\omega)$ in (2) means that the $E_{i}(\mathbf{R}, \omega)$ is supposed as local field relative to the nanocomposite film but external field relative to a single nanoparticle. Other words, it was supposed that intraparticle interactions ("1" in Fig.2)) are taken into account by choosing particle susceptibility $X_{j l}^{(p)}(\omega)$, but interparticle interactions ("2" in Fig.2)) form the local field in (2).

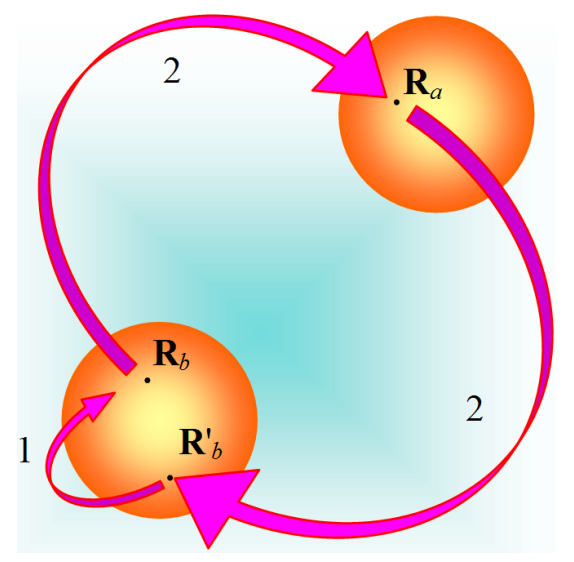

Figure 2. The sketch of contribution of self-action fields via the matrix (1) and via interactions with other particles (2).

The inclusion particles are assumed to be located at the average distances of order or more than linear dimensions of the particles from each other. As it was shown in [33], in this case, the particles are polarized like non-point particles with a shape but interacting as point-like objects. In this case, the equation (2) for self-consistent field has a form:

$$
\begin{aligned}
& E_{i}(\mathbf{r}, z, \omega)=E_{i}^{(0)}(\mathbf{r}, z, \omega)-\left(k_{0}^{2} / d\right) \\
& \quad \times\left\langle\int_{-d / 2}^{d / 2} d z^{\prime} \sum_{a=1}^{N\left(z^{\prime}\right)} G_{i j}^{(2)}\left(\mathbf{r}-\mathbf{r}_{a}, z, z^{\prime}, \omega\right) \tilde{\mathbf{X}}_{j l}^{(p)}(\omega) E_{l}\left(\mathbf{r}_{a}, z^{\prime}, \omega\right)\right\rangle_{S}
\end{aligned}
$$

Here, $\tilde{X}_{j l}^{(p)}(\omega)=V_{a} X_{j l}^{(p)}(\omega)$ is the polarizability of inclusion particle, $V_{a}$ is a volume of particle, and $S$ is the area of the film surface. To differ the in-plane and out-of-plane coordinates, the following notations are used for radius vector

$$
\mathbf{R}=(\mathbf{r}, z) \text {. Notation }
$$

$$
\langle\ldots\rangle_{S}=\left(1 / S^{N(z)}\right) \prod_{a=1}^{N(z)} \int_{S} d \mathbf{r}_{a}(\ldots)
$$

means averaging over the positions of inclusion particles, assuming that $N(z)$ inclusion particles are distributed homogeneously over the film plane at any fixed coordinate $z$. This averaging implies that spatial distribution of a given particle is independent on locations of the other particles. In this connection, we should take into account nonpointness of the particles, which should be reflected in a constraint on the particle concentration. Namely, the volume fraction of the inclusion particles inside the nanocomposite should not exceed the volume $V$ of the entire system, namely, $N_{p} V_{a}<V$. Note that the in-plane averaging is depended on the plane in which the averaging is performed. Then, number of the particles in $z$ th layer is the function of $z$, and the function $N(z) / S$ is in fact the distribution function of inclusions.

The problem of finding the local field at any point inside the system and calculating the optical response to the external field is very similar to the calculation of the effective susceptibility for nanosystems considered in [31].

The averaging over the inclusion particles distribution of (3) gives the Fourier transform (in the plane of the film surface) of self-consistent local field in the system under consideration in the form (see details of the calculations in [31])

$$
E_{i}\left(\mathbf{k}_{\|}, z, \omega\right)=\Xi_{i j}\left(\mathbf{k}_{\|}, z, \omega\right) E_{j}^{(\mathrm{ext})}\left(\mathbf{k}_{\|}, z, \omega\right)
$$

with the "effective" local field factor playing a role of "effective" dielectric function of nanocomposite thin film

$$
\Xi_{i j}\left(\mathbf{k}_{\|}, z, \omega\right)=\mathbf{X}_{i l}^{(f)}\left(\mathbf{k}_{\|}, z, \omega\right) L_{l j}^{(0)}\left(\mathbf{k}_{\|}, z, \omega\right)
$$

the effective susceptibility of the film

$$
\mathbf{X}_{i l}^{(f)}=\left[\delta_{l i}+n(z) V_{a} \frac{k_{0}^{2}}{d} \int_{-d / 2}^{d / 2} d z^{\prime} G_{l q}^{(2)}\left(\mathbf{k}_{\|}, z^{\prime}, z, \omega\right) \mathbf{X}_{q i}^{(p)}(\omega)\right]^{-1}
$$

and the local field factor

$$
L_{i j}^{(0)}\left(\mathbf{k}_{\|}, z, \omega\right)=G_{i l}^{(2,1)}\left(\mathbf{k}_{\|}, z, z^{\prime}, \omega\right)\left[D_{j l}\left(\mathbf{k}_{\|}, z, z^{\prime}, \omega\right)\right]^{-1}
$$

where $E_{j}^{(\text {ext })}\left(\mathbf{k}_{\|}, z, \omega\right)$ is the Fourier transform of the external (with respect to the entire system) electric field, $G_{i j}^{(2,1)}\left(\mathbf{k}_{\|}, z, z^{\prime}, \omega\right)$ is the Green function [32] describing the field at the cross-section $z$ (inside the film $-d / 2 \leq z \leq d / 2$ ) induced by the source point located at cross-section $z^{\prime}$ in the substrate $z^{\prime}<-d / 2$ (see Fig. 1), and $D_{j l}\left(\mathbf{k}_{\|}, z, z^{\prime}, \omega\right)$ is the complete bulk response function [32]. $n(z)=N(z) / S$. Here and hereafter, the element of the inverse of a matrix $\left(A^{-1}\right)_{i j}$ is denoted by $\left[A_{j i}\right]^{-1}$. Note that the film is irradiated from the side of substrate unlike [34], where the irradiation was carried out from the side of the environment. 


\section{Optical Absorption by Thin Nanocomposite Film with Different Distributions of Inclusions along Film Thickness}

To analyze the optical absorption by nanocomposite film, one should write the dissipative function of a system. To simplify the problem, we can suppose that the probing beam is monochromatic, linear polarized and directed normally to the surface of a film (the wave vector $\mathbf{k}_{\|}$equals to zero). The dissipative function having the sense of averaged energy absorbed by the unit volume of the system per time unit is the cycle-averaged Joule heating in the case under consideration written as

$$
Q(\omega)=\frac{1}{4 d} \int_{-d / 2}^{d / 2} d z\left[E_{i}^{*}(z, \omega) J_{i}(z, \omega)+E_{i}(z, \omega) J_{i}^{*}(z, \omega)\right]
$$

Here, $E_{i}(z, \omega)$ and $J_{i}(z, \omega)$ are the local field and local current inside the film, respectively. Taking into account

$$
J_{i}(z, \omega)=-i \omega \varepsilon_{0} f(z) \mathbf{X}_{i j}^{(p)}(\omega) E_{j}(z, \omega)
$$

with the absorbing volume fraction of inclusions in the film per unit area of cross-section $z$ as $f(z)=n(z) V_{a} / d$, and neglecting the absorption in matrix, one can write dissipation function in the form

$$
Q(\omega)=\frac{\omega \varepsilon_{0}}{2 d} \operatorname{Im} X^{(p)}(\omega) \sum_{i, l} \int_{-d / 2}^{d / 2} d z f(z)\left|\Xi_{i l}(z, \omega)\right|^{2}\left|E_{l}^{(\mathrm{ex} t)}\right|^{2}
$$

Here, $\Xi_{i j}(z, \omega)=\Xi_{i j}\left(\mathbf{k}_{\|}=0, z, \omega\right)$ and for symmetrical spherical particles $\mathrm{X}_{i j}^{(p)}(\omega)=\mathrm{X}^{(p)}(\omega) \delta_{i j}$.

Using Cartesian coordinate system where incidence plane coincides with the $x z$ plane and film plane coincides with the $x y$ plane of coordinate system, and taking into account an assumption that incident beam is directed normally to the surface of the film, we can see from [32] that only one component (the $y y$-component) of the Green function has to be taken into account.

The absorption profile can be written as

$$
\begin{gathered}
I(\omega)=\frac{\hbar Q(\omega)}{\varepsilon_{0}\left|E_{y}^{e x t}(\omega)\right|^{2}}, \\
I(\omega)=\frac{\hbar \omega}{2 d} \operatorname{Im} X^{(p)} \int_{-d / 2}^{d / 2} d z f(z)\left|\Xi_{y y}(z, \omega)\right|^{2} .
\end{gathered}
$$

For discussion of the plasmonic resonances of coated spheres, we consider the extension of Mie's theory on a sphere (core radius $a_{1}$ ) coated with one shell of different thickness in the Rayleigh approximation, so that only the TM dipole mode contributes to the absorption [29]. In this case, the effective susceptibility for single spherical particle with shell is found [35] to be as

$$
\mathrm{X}^{(p)}=4 \pi a_{2}^{3} \frac{\left(\varepsilon_{2}-\varepsilon_{m}\right)\left(\varepsilon_{1}+2 \varepsilon_{2}\right)+f_{1}\left(\varepsilon_{1}-\varepsilon_{2}\right)\left(\varepsilon_{m}+2 \varepsilon_{2}\right)}{\left(\varepsilon_{2}+2 \varepsilon_{m}\right)\left(\varepsilon_{1}+2 \varepsilon_{2}\right)+f_{1}\left(2 \varepsilon_{2}-2 \varepsilon_{m}\right)\left(\varepsilon_{1}-\varepsilon_{2}\right)}
$$

Here, $\varepsilon_{m}$ is the dielectric constant of the matrix, $\varepsilon_{1}$ and $\varepsilon_{2}$ are the dielectric constants of a spherical particle of radius $a_{1}$ and the shell, respectively. The radius of a spherical particle with a shell is $a_{2}$.

$$
f_{1}=a_{1}^{3} / a_{2}^{3}
$$

By use of (13) with (14), (15) and (6)-(8) we calculate the absorption profiles of nanocomposite films with embedded GNPs with shell in Teflon matrix with $\varepsilon_{\mathrm{m}}=1.27$. The experimental values of optical constants of $\mathrm{Au}$ are derived from [36].

The absorption profiles have been numerically calculated for 300-nm-thickness nanocomposite films with three different distributions of identical inclusions across thickness inside the different film:

Inclusion particles are distributed homogeneously across the film thickness and layer concentration of inclusion particles is constant

$$
n_{0}(z)=n_{0}=\text { const }, \quad-1 / 2 \leq z / d \leq 1 / 2 .
$$

The layer concentration of inclusion particles is linear dependent on coordinate $z$ :

$$
n_{1}(z)=n_{0}(1+2 z / d), \quad-1 / 2 \leq z / d \leq 1 / 2,
$$

here, the concentration of inclusion particles is minimal at the irradiated surface $z=-d / 2$; and

$$
n_{2}(z)=n_{0}(1-2 z / d), \quad-1 / 2 \leq z / d \leq 1 / 2,
$$

here, the concentration of inclusion particles is maximal at the irradiated surface $z=-d / 2$.

\section{Nanocomposite Films in Sensorics}

The electrodynamic interaction between elementary excitations inside the inclusions and an electromagnetic field leads to hybridized states. The excitations of these states are called plasmon resonances. Localized plasmons play an essential role in the absorption profiles formation. Numerous important applications of localized plasmons resonance phenomenon were demonstrated in recent years. For example, the absorbance of gold colloids is sensitive to the refractive index of the surrounding solvent [37]. Several experimental works related to influence of nanoparticle (NP) systems parameters (e.g. shape, size and spacing) on their extinction properties [38-40]. It means that modified nanoparticles inside the matrix, through which the biomolecules are able to pass, can play a role of sensitive device for sensing both the biomolecules concentration in the bioliquid and biospecific interactions (interaction antigen-antibody type [41]). Indeed, let imagine that the nanoparticles embedded in a thin film of 
wadding type are modified with the pairs of immunoglobuline IgG-protein $\mathrm{G}$. Then each of the inclusion particles can be considered as the coated metal particle with biomolecular layer (Fig. 3). When this nanocomposite thin film puts into in specific bioliquid, due to the wadding type of its matrix the biospecific interaction can occur, the anti-Ig molecules can accede to suitable parts of molecules Ig. As a result, the characteristics of nanoparticle shell will be changed; at least, its thickness will be increased that in its turn should influence the optical properties of entire nanocomposite film.

Note that molecules of $\mathrm{IgG}$ have rather large linear dimensions (its length is about $20 \mathrm{~nm}$ ). Then, to use the local plasmon resonance for sensing, one needs to provide the molecules transportation from external solution to the nanoparticles inside the film-matrix. It means that the matrix should be fabricated from a fibrous material. Fortunately, the Teflon film fabricated with vacuum deposition often has a structure of fibrous material [42]. The average distances between the Teflon fibers are about $100 \mathrm{~nm}$. Then, there is a possibility for the rather large bio-molecules to penetrate inside the film.
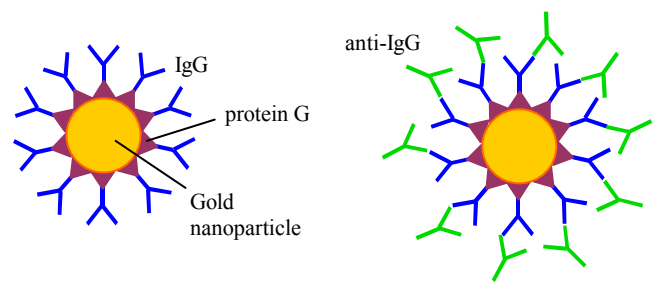

Figure 3. Sketch of modified nanoparticle (left) which is covered by anti-immunoglobuline (right).

In nanocomposite film the plasmon resonant frequency failed to be defined by poles of effective susceptibility. To find this frequency, the dissipative function of entire nanocomposite system should be analyzed.

A shell on metal NP should modify the optical properties of the nanocomposite. To find an effect of the shell we have calculated the dissipative function of film with modified NP. Pronounced peaks in obtained absorption profiles are obviously caused by plasmon excitations.

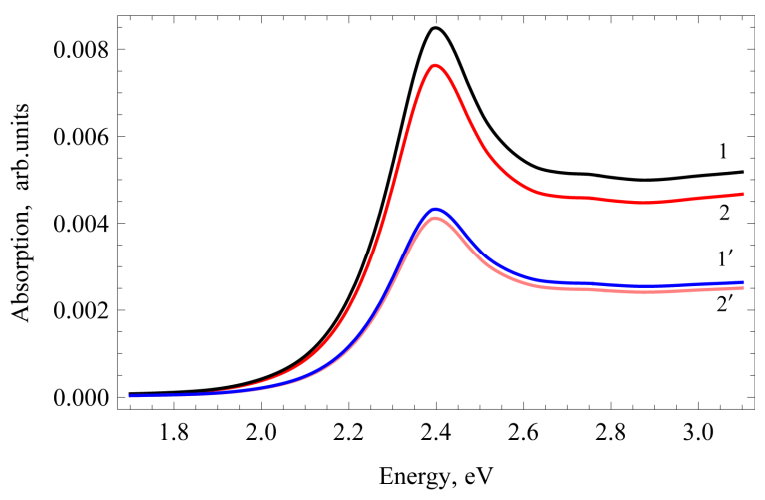

Figure 4. The changing of absorption profile of nanocomposite thin $(300 \mathrm{~nm})$ film when layer of anti-IgG binds to the surface of inclusion. Curve 1 corresponds to initial modified inclusions; curve 2 corresponds to the film treated with solution of anti-IgG $(f=0.001)$. (Curves $1^{\prime}$ and $2^{\prime}$ correspond to lower gold volume fraction, $f=0.0005$ ).
We calculated the absorption profiles for nanocomposite 300-nm-thickness film with embedded gold spheres of 8-nm radius, modified by molecular layer of rather big molecules (e.g. IgG-proteinG complexes) having 27-nm dimension. We took into account the absorption only by gold fraction of nanoparticles neglecting absorption in shells and matrix. After binding of molecules anti-IgG to the NP surface the shell thickness becomes $54 \mathrm{~nm}$. Then, the absorption profiles should be changed as one can see in Fig. 4. As shown, the biospecific interaction leads to absorption profile changing. The changing is increased while increasing the inclusions concentration. Note the increasing of shell thickness leads to decreasing of absorption peak in a case of thick shell relative to NP core and no absorbing shells.

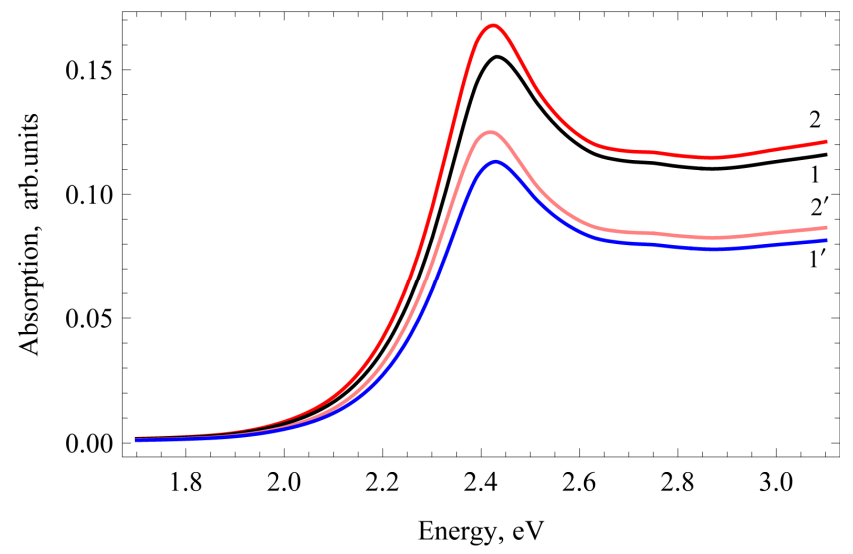

Figure 5. The changing of absorption profile of nanocomposite thin film when layer of "small" molecules binds to the surface of inclusion. Curve 1 corresponds to initial modified inclusions; curve 2 corresponds to the film treated with solution of "small" complementary molecules $(f=0.03)$. (Curves $I^{\prime}$ and $2^{\prime}$ correspond to lower gold volume fraction $\left.f=0.02\right)$.

Because the complementary interactions can be also observed for pairs of rather small molecules [43], the similar calculations were performed for the case of "small" molecular shells. The absorption profiles are calculated for nanocomposite 300-nm-thickness film with embedded gold spheres of 8-nm radius, modified by molecular layer of small molecules having 5-nm dimension. After binding of complementary molecules to the NP surface the shell thickness becomes $10 \mathrm{~nm}$. Then, the absorption profiles should be changed as one can see in Fig. 5. Unlike the previous case (shown in Fig. 4), the increasing of shell thickness leads to increasing of absorption peak even in the case of no absorbing shells. By observing the corresponding absorption profile change one can also detect whether the bio-specific reaction occurred.

The dependencies of the absorption profile for the nanocomposite thin film on the thickness of shell of modified GNPs and on distribution of inclusions across thickness of film are analyzed. The absorption profiles for three values of the film thickness are presented in Fig. 6. Note that in visible range the average absorption of 100-nm-thickness film is larger comparing to 200-nm-thickness and 300-nm-thickness films with the same average volume fraction of the same embedded modified nanospheres. 
We have also calculated the influence of the distribution of gold spheres of 8-nm radius, modified by molecular layer of rather big molecules (e.g. IgG-proteinG complexes) having 27-nm dimension, on the absorption profile of nanocomposite films. The volume fraction of gold was supposed as $f=0.001$. The results obtained for three distributions of embedded particles (16), (17) and (18) showed that a replacing of the homogeneous distribution with the linear one leads to slight changing of absorption profile in visible range. Therefore one can assume that in visible range the absorption profiles are practically independent on the inclusions distribution across the film thickness in the case of very small value of volume fraction $f$.

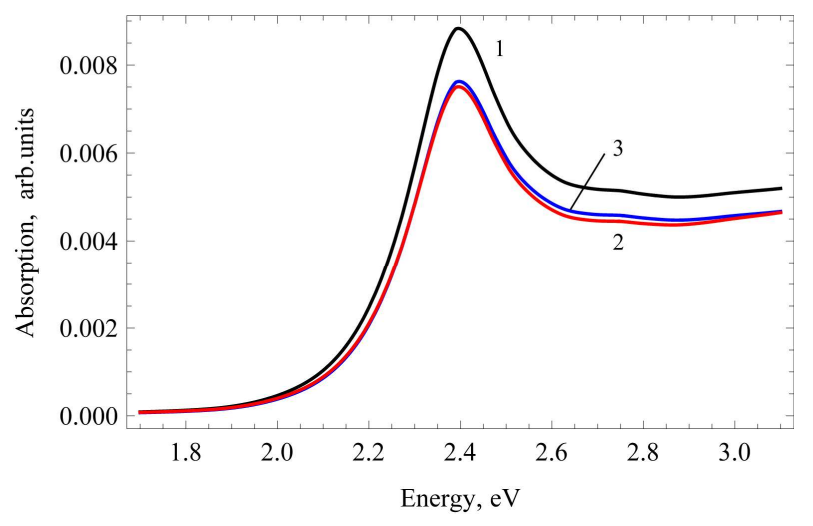

Figure 6. The absorption profile for nanocomposite film treated with solution of anti-IgG with homogeneous distribution of embedded particles $(f=0.001)$ for three different thicknesses of the film: Curve 1 (black) corresponds to 100 $\mathrm{nm}$, curve 2 (red) corresponds to $200 \mathrm{~nm}$, and curve 3 (blue) corresponds to $300 \mathrm{~nm}$.

\section{Conclusions}

The self-consistent method of calculating the optical response is applied to the nanocomposite film with inhomogeneous distribution of modified inclusions across the film thickness.

Obtaining of the nanocomposite films with the certain distribution of embedded particles across the thickness of the film can require additional technological efforts. However, as being shown, in the case of the small gold volume fraction in the film the changing of absorption profile with change of distributions of embedded particles is negligible. This allows applying technology of the simplest possible distribution of embedded nanoparticles with account for needed averaged gold volume fraction in the films.

The localized plasmon resonance exposed in absorption spectra can be used for sensing the change in shell thickness of modified gold nanoparticles caused for example by biospecific interactions at the surface of nanoinclusions.

Increase in the nanoparticle shell thickness results in absorption profile change. Change trend is obtained in a case for no absorbing "big" and "small" molecular shells. Taking this into account, one can detect whether biospecific reaction took place by observing the corresponding absorption profile change.

Finally, we note that the case of normal incidence of probing light has been considered for reasons of simple geometry for implementation of biosensor device. Preliminary analysis (results are not included in this work) showed that an use of the probing light under certain angles to film surface can result in strengthened effect of the increase in the shell thickness of the embedded particles and thereby enhances the sensitivity of the device.

\section{References}

[1] H.-W. Fink and C. Schönenberger, "Electrical conduction through DNA molecules," Nature, vol. 398, no. 6726, pp. 407-410, 1999.

[2] D. Porath, A. Bezryadin, S. de Vries, and C. Dekker, "Direct measurement of electrical transport through DNA molecules," Nature, vol. 403, no. 6770, pp. 635-638, 2000.

[3] O. V. Salata, "Applications of nanoparticles in biology and medicine," Journal of Nanobiotechnology, vol. 2, Article ID 3, 6 pages, 2004.

[4] H.-E. Schaefer, Nanoscience: The Science of the Small in Physics, Engineering, Chemistry, Biology and Medicine, Springer, Berlin, 2010.

[5] Yu. A. Berlin, A. L. Burin, and M. A. Ratner, "DNA as a molecular wire," Superlattices and Microstructures, vol. 28, no. 4, pp. 241-252, 2000.

[6] S. Brasselet, "Polarization-resolved nonlinear microscopy: application to structural molecular and biological imaging," Advances in Optics and Photonics, vol. 3, no. 3, pp. 205-271, 2011.

[7] J. P. Jagtap, T. H. Jadhav, and D. Utpal, "Physical properties of virus causing cotton mosaic disease," Scientific Journal of Crop Science, vol. 1, no. 1, pp. 9-15, 2012.

[8] J. L. Elechiguerra, J. L. Burt, J. R. Morones, A. Camacho-Bragado et al., "Interaction of silver nanoparticles with HIV-1," Journal of Nanobiotechnology, vol. 3, Article ID 6, 10 pages, 2005.

[9] T. A. Delchar, Physics in Medical Diagnosis, Springer, Berlin, 1997.

[10] D. W. Kang, X. P. Hao, X. Z. Li, L. B. Li, and S. J. Xie, "Spin polarized current through Cu-DNA modulated by a gate voltage," Applied Physics Letters, vol. 102, no. 7, pp. 072410(1-4), 2013.

[11] B. Göhler, V. Hamelbeck, T. Z. Markus et al., "Spin selectivity in electron transmission through self-assembled monolayers of double-stranded DNA," Science, vol. 331, no. 6019, pp. 894-897, 2011.

[12] A. A. Eremko and V. M. Loktev, "Spin sensitive electron transmission through helical potentials," Physical Review B, vol. 88, no. 16, pp. 165409, 2013.

[13] O. Grynko, V. Lozovski, O. Ozerov, S. Repetskii, O. Tretyak, and I. Vyshyvana, "Electron spin-polarizer on the base of DNA array," 2014 IEEE 34th International Scientific Conference on Electronics and Nanotechnology, ELNANO 2014 Conference Proceedings 6873921, pp. 250-253. 
[14] Surface Polaritons: Electromagnetic Waves at Surfaces and Interfaces, V. M. Agranovich and D. L. Mills, Eds. Amsterdam, North-Holland, 1982.

[15] J. Davies, "Surface plasmon resonance — the technique and its applications to biomedical processes," Nanobiology, vol. 3, pp. $5-16,1994$.

[16] J. Homola, "Present and future of surface plasmon resonance biosensors," Analytical and Bioanalytical Chemistry, vol. 377, no. 3, pp. 528-539, 2003.

[17] N. F. Starodub, T. L. Dibrova, Yu. M. Shyrshov, and K. V. Kostyukevich, "Development of the myoglobin sensor based on the surface plasmon resonance," Ukrains'kyi Biokhimichnyi Zhurnal, vol. 71, no. 2, pp. 33-37, 1999.

[18] Optical Sensors: Industrial Enviromental and Diagnostic Applications, R. Narayanaswamy and O. S. Wolfbeis, Eds. Springer, Berlin, 2004.

[19] C. Y. Yao and W. L. Fu, "Biosensors for hepatitis B virus detection," World Journal of Gastroenterology, vol. 20, no. 35, pp. 12485-12492, 2014.

[20] D. Lepage and J. J. Dubowski, "Miniaturized quantum semiconductor surface plasmon resonance platform for detection of biological molecules," Biosensors, vol. 3, no. 2, pp. 201-210, 2013.

[21] D. Hu, S. R. Fry, J. X. Huang et al., "Comparison of surface plasmon resonance, resonant waveguide grating biosensing and enzyme linked immunosorbent assay (ELISA) in the evaluation of a Dengue Virus immunoassay," Biosensors, vol. 3, no. 3, pp. 297-311, 2013

[22] M. Holzinger, A. Le Goff, and S. Cosnier, "Nanomaterials for biosensing applications: a review," Frontiers in Chemistry, vol. 2, Article ID 63, 10 pages, 2014.

[23] N. L. Rosi and C. A. Mirkin, "Nanostructures in biodiagnostics," Chemical Reviews, vol. 105, no. 4, pp. 1547-1562, 2005.

[24] C. Hüttl, C. Hettrich, R. Miller et al., "Self-assembled peptide amphiphiles function as multivalent binder with increased hemagglutinin affinity," BMC Biotechnology, vol. 13, Article ID 51, 10 pages, 2013.

[25] V. Chegel, Yu. Chegel, M. D. Guiver, A. Lopatynskyi, O. Lopatynska, and V. Lozovski, "3D-quantification of biomolecular covers using surface plasmon-polariton resonance experiment," Sensors and Actuators B, vol. 134, no. 1, pp. 66-71, 2008.

[26] J. Homola, S. S. Yee, and G. Gauglitz, "Surface plasmon resonance sensors: review", Sensors and Actuators B, vol. 54, pp. 3-15, 1999.

[27] J. Homola, "Surface plasmon resonance sensors for detection of chemical and biological species", Chemical Reviews, vol. 108, pp. 462-493, 2008.

[28] V. Lozovski, "The effective susceptibility concept in the electrodynamics of nano-systems," Journal of Computational and Theoretical Nanoscience, vol. 7, no. 10, pp. 2077-2093, 2010 .
[29] M. Quinten, Optical Properties of Nanoparticle Systems: Mie and Beyond, Wiley-VCH Verlag \& Co. KGaA, Germany, 2011.

[30] N. G. Khlebtsov, "Optics and biophotonics of nanoparticles with a plasmon resonance", Quantum Electronics, vol. 38, no. 6, pp. 504-529, 2008.

[31] V. Lozovski, M. Razumova, and G. Strilchuk, "Self-consistent approach to calculation of the optical response and absorption profiles of thin nanocomposite films", Plasmonics, 2015, in press.

[32] M. L. Bah, A. Akjouj, and L. Dobrzynski, "Response functions in layered dielectric media," Surface Science Reports, vol. 16, pp. 97-131, 1992.

[33] I. Iezhokin, O. Keller, and V. Lozovski, "Induced light emission from quantum dots: the directional near-field pattern," Journal of Computational and Theoretical Nanoscience, vol. 7, no. 1, pp. 281-288, 2010.

[34] V. Lozovski and M. Razumova, "The local field effects in optical response of nanocomposite thin films. An implementation in sensorics of biospecific interactions," 2015 IEEE 35th International Conference on Electronics and Nanotechnology (ELNANO-2015), Conference Proceedings, pp. 333-336, 2015.

[35] L. G. Grechko, N. G. Skoda, and S. V. Shostak, "Effective dielectric permittivity of matrix disperse systems with two-layer inclusion", Ukrainian Journal of Physics, vol. 47, no. 7, pp. 694-698, 2002.

[36] P. B. Johnson and R. W. Christy, "Optical constants of noble metals,” Physical Review B, vol. 6, pp. 4370-4379, 1972.

[37] E. F. Venger, A. V. Goncharenko, M. L. Dmitruk, Optics of Small Particles and Composite Media, Naukova dumka, Kyiv, 2009.

[38] J. J. Mock, D. R. Smith and S. Schultz, "Local refractive index dependence of plasmon resonance spectra from individual nanoparticles," Nano Letters, vol. 3, no. 4, pp. 485-491, 2003.

[39] J. J. Mock, M. Barbic, D. R. Smith, D. A. Schultz and S. Schultz, "Shape effects in plasmon resonance of individual colloidal silver nanoparticles", Journal of Chemical Physics, vol. 116, pp. 6755-6759, 2002.

[40] K. H. Su, Q. H. Wei, X. Zhang, et al., "Interparticle coupling effects on plasmon resonances of nanogold particles", Nano Letters, vol. 3, no. 8, pp. 1087-1090, 2003.

[41] C. A. Janeway, P. Travers, M. Walport, and M.Shlomchik, Immunobiology. 6th Edition. Garland Publishing, New York, 2001.

[42] V. Lozovski, S. Khihlovski, K. Grytsenko, V. Ksianzou, S. Schrader, and G. Strilchuk, "Modelling of optical absorption of three-component nanocomposite thin films", Physica Status Solidi (b), vol. 247, pp. 2244-2251, 2010.

[43] P. F. Dillon, R. S. Root-Bernstein, C. M. Lieder, "Molecular shielding of electric field complex dissociation", Biophysical journal, vol. 90, no. 4, pp. 1432-1438, 2006. 